\title{
Esophageal Polyp Lesion in a Patient with Globus Pharyngeus Compliant
}

\author{
Amirhossein Boghratian ${ }^{1}$, Nasser Rakhshani ${ }^{2}$, Jamshid Vafaeimanesh ${ }^{3,4, *}$
}

1. Colorectal Research Center, Iran University of Medical Sciences, Tehran, Iran

2. Gastrointestinal and Liver Diseases Research Center, Iran University of Medical Sciences, Tehran, Iran

3. Clinical Research Development Center, Qom University of Medical Sciences, Qom, Islamic Republic of Iran

4. Associate Professor, Gastroenterology and Hepatology Disease Research Center, Qom University of Medical Sciences, Qom, Islamic Republic of Iran

\section{* Corresponding Author:}

Jamshid Vafaeimanesh, MD

Gastroenterology and Hepatology Disease Research Center, Shahid Beheshti Hospital, Shahid Beheshti Boulevard, Qom, Iran Tel: + 982536122949

Fax: + 982536122945

Email: jvafaeemanesh@yahoo.com

Received: 17 Jul. 2019

Accepted: 11 Nov. 2019

\section{Please cite this paper as:}

Boghratian AH, Rakhshani N, Vafaeimanesh J. Esophageal Polyp Lesion in a Patient with Globus Pharyngeus Compliant. Middle East J Dig Dis 2020;12:52-54. doi: 10.15171/mejdd.2020.165.

A 43-year-old woman referred to our gastroenterology clinic with dyspepsia and heartburn. In her medical history, she complained of epigastric pain and globus signs and took anti-acids periodically. She did not have weight loss and there was no family history of gastrointestinal malignancy. She had microcytic anemia, which was treated with daily ferrous ( 2 pills, $50 \mathrm{mg}$ elemental iron). During the physical examination, only tenderness at epigastrium was noted. Clinical signs including globus Pharyngeus did not improve in spite of two months of treatment with proton pump inhibitors (PPI). Considering the patient's age, upper gastrointestinal endoscopy was done and a small polyp lesion (5 $\mathrm{mm}$ ) was seen in the upper esophagus (figure 1). A biopsy sample was taken and $\mathrm{H} \& \mathrm{E}$ staining is shown in figures 2,3 , and 4 .

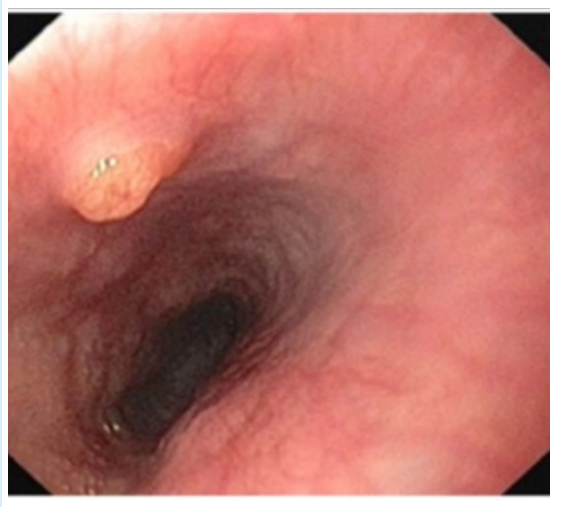

Fig.1: Endoscopic view: smallpolyplesion $(5 \mathrm{~mm})$ was seen in the upper esophagus

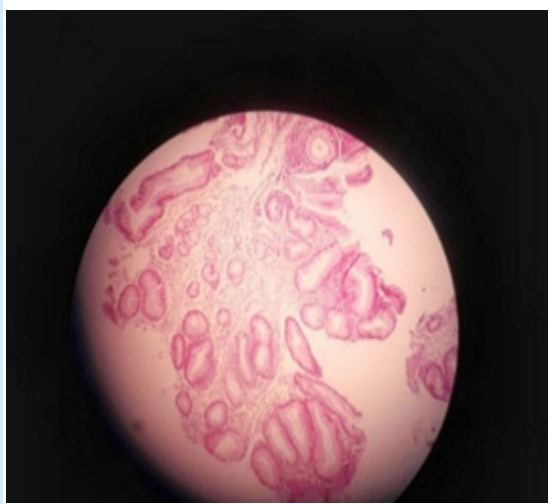

Fig.3: H\&E slides

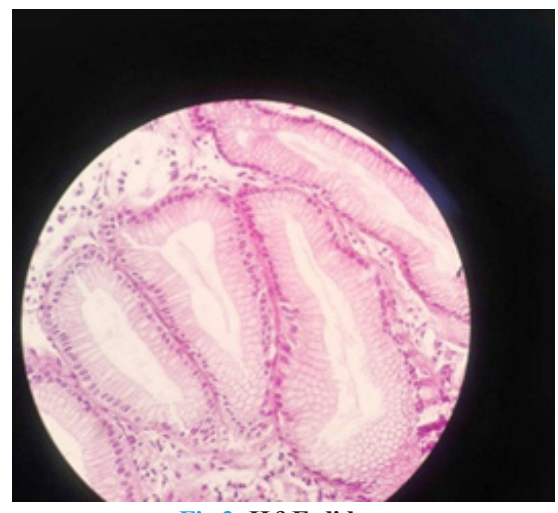

Fig.2: H\&E slides

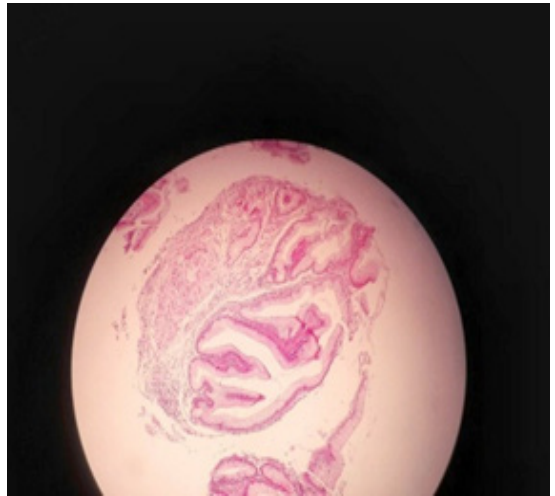

Fig.4: H\&E slides

2020 The Author(s). This work is published by Middle East Journal of Digestive Diseaes as an open access org/licenses/by-nc/4.0/). Non-commercial uses of the work are permitted, provided the original work is properly cited.
article distributed under the terms of the Creative Commons Attribution License (https:/creativecommons. 
What is your diagnosis?

Answer:

\section{Inlet patch}

The inlet patch is not very prevalent. Radiographic evidence shows the inlet patch prevalence as $2.4 \%$ at the upper esophagus. ${ }^{1}$ In endoscopic observation, its prevalence was reported as $0.1-13.8 \%{ }^{2}$

The lesion is seen more in men $(3.1 \%)$ than women $(0.6 \%)$. In most cases, the patch is reported as flat. In recent reports, there is limited number of polyps that were formed at the neck of the esophagus ${ }^{1-3}$ and even in one case, a large polyp was formed, which caused severe dysphasia. ${ }^{4}$

This lesion emerges as a polyp in affected patients. It seems that cases of inlet patch polyp have higher clinical importance and they may be more prone to malignancy. No certain risk factors have been reported for malignancy in patients with inlet patch and as a result, there is no consensus on the development of malignancy in these patients, although it is possible and interestingly has been reported in some articles. ${ }^{5}$

However, bypass samples should be examined in terms of dysplasia and follow-up and repeated bypass is suggested when intestinal metaplasia or dysplasia is seen. Although development to malignancy is rare, expert endoscopists should be aware of this potential risk. Inlet patch may be recognized in patients who are exposed to more risk for the development of proximal esophagus adenocarcinomas; however, this relationship and the necessity of screening requires further studies. ${ }^{6}$

As it was discussed, most cases of inlet patch are found by chance and without making any clinical signs. Thus, total treatment is done when outcomes emerge. Some studies have shown that acid is released from the inlet patch. ${ }^{3-7}$ Proton pomp $\left(\mathrm{H}^{+}, \mathrm{K}^{+}\right.$, -ATPase) influence at the inlet patch has been shown by immunohistochemistry. ${ }^{8}$ PPI influence in some studies supports the theory that acid released from proton pomp in patients with inlet patch may cause the emergence of globuspharyngeus signs. In one study, the inlet patch has been known as one of the main causes of globus pharyngeus. ${ }^{9}$

Our patient also had globuspharyngeus signs and responded to acid inhibition treatment with PPI.
The polyp was seen in $20 \mathrm{~cm}$ from the incisor teeth in endoscopy. H\&E staining showed different magnification from the lesion, which demarcated as an antral type gastric mucosa with regenerative changes. No intestinal metaplasia or dysplasia was seen. Test for helicobacter pylori was negative in Giemsa staining and the lesion was compatible with inlet patch. However, further studies are needed to show the efficiency of PPI for patients with globuspharyngeus signs and inlet patch. Recently, Argon plasma coagulation and radiofrequency degradation have been useful for cervical inlet patch. ${ }^{10-11}$ Moreover, recent studies have shown that the treatment with radiofrequency ablation (RFA) can be useful in returning the abnormal mucus in the inlet patch to normal level. ${ }^{12}$

\section{CONCLUSION}

This report showed that the inlet patch should be considered as a differential diagnosis for globuspharyngeus signs and this disorder can even emerge as a polyp lesion. It should be considered that the inlet patch may be neglected most of the time during upper gastrointestinal endoscopy. It is important to develop a systematic routine to reduce the possibility of missing any area. The diagnosis of the inlet patch is confirmed by endoscopy and taking a biopsy sample. The lesion is seen more often by endoscopists whose custom is to withdraw the scope very slowly through the upper sphincter in order to inspect the arytenoids and vocal cords.

\section{ETHICAL APPROVAL}

There is nothing to be declared. (Informed consent was taken from the patient)

\section{CONFLICT OF INTEREST}

The authors declare no conflict of interest related to this work.

\section{REFERENCES}

1. Takeji H, Ueno J, Nishitani H. Ectopic gastric mucosa in the upper esophagus: prevalence and radiologic findings. AJR Am J Roentgenol 1995;164:901-4. doi: 10.2214/ ajr.164.4.7726045

2. Maconi G, Pace F, Vago L, Carsana L, Bargiggia S, Bianchi Porro G. Prevalence and clinical features of heterotopic gastric mucosa in the upper oesophagus (inlet patch).Eur J Gastroenterol Hepatol 2000;12:745-9. doi: 10.1097/00042737200012070-00005. 
3. Korkut E, Bektaş M, Alkan M, Ustün Y, Meco C, Ozden $\mathrm{A}$, et al. Esophageal motility and 24-h $\mathrm{pH}$ profiles of patients with heterotopic gastric mucosa in the cervical esophagus. Eur J Intern Med 2010;21:21-4.doi: 10.1016/j. ejim.2009.10.009.

4. Rana SS, Panda N, Sinha SK, Nada R, Nagi B, Bhasin DK.Heterotopic gastric mucosa in the upper esophagus manifesting as a polypoidal mass causing dysphagia.Gastrointest Endosc 2006;63:185-7.doi: 10.1016/j.gie.2005.07.042.

5. Alagozlu H, Ergun M, Cindoruk M, Unal S, Dumlu S, Poyraz A, et al. The rare presentations of a large polyp and an esophageal carcinoma in heterotropic gastric mucosa: a case series. J Med Case Rep 2007;1:127. doi:10.1186/1752-1947-1-127.

6. Riddiough GE, Hornby ST, Asadi K, Aly A.Gastric adenocarinoma of the upper oesophagus: A literature review and case report. Int J Surg Case Rep 2017;30:205-14. doi: 10.1016/j.ijscr.2016.11.014.

7. Galan AR, Katzka DA, Castell DO. Acid secretion from an esophageal inlet patch demonstrated by ambulatory pH monitoring. Gastroenterology 1998;115:1574-6. doi:10.1016/s0016-5085(98)70038-1.

8. Yamada T, Tsuji A, Onoue S, Kaneko M, Tanioka F, Osawa $\mathrm{S}$, et al. Acid suppressive therapy improved symptoms due to circumferential cervical inlet patch with proton pumps (H+/K+-ATPase). World J Clin Cases 2017 16;5:403-6. doi: 10.12998/wjcc.v5.i11.403.

9. Chong VH. Cervical inlet patch: an important cause of Globus pharyngeus. Eur Arch Otorhinolaryngol 2018;275:3101-2. doi: 10.1007/s00405-018-5132-z.

10. Meining A, Bajbouj M, Preeg M, Reichenberger J, Kassem AM, Huber W, et al. Argon plasma ablation of gastric inlet patches in the cervical esophagus may alleviate globus sensation: a pilot trial. Endoscopy 2006;38:566-70. doi:10.1055/s-2006-925362.

11. Dunn JM, Sui G, Anggiansah A, Wong T. Radiofrequency ablation of symptomatic cervical inlet patch using a through-the-scope device: a pilot study. Gastrointest Endosc 2016;84:1022-1026.e2. doi: 10.1016/j. gie.2016.06.037. Epub 2016 Jul 1.

12. Rusu R, Ishaq S, Wong T, Dunn JM. Cervical inlet patch: new insights into diagnosis and endoscopic therapy. Frontline Gastroenterol 2018;9:214-220. doi: 10.1136/ flgastro-2017-100855. 